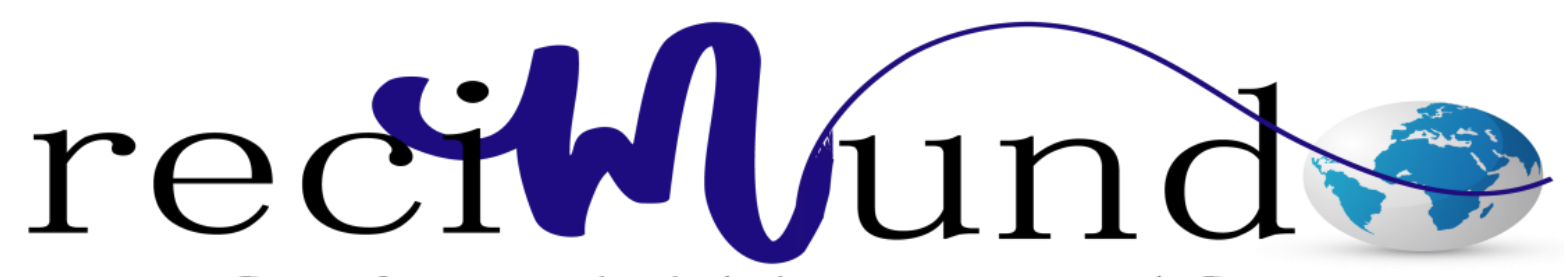

Revista Cientifica Mundo de la Investigación y el Conocimiento

\author{
Álvaro Miguel González Ortiz a; Vanessa Jomaira Veintimilla Abril ${ }^{\text {b; }}$ Marisela \\ Susana Villarreal Salazar ${ }^{c}$
}

Nivel de satisfacción del uso de prótesis totales bimaxilares en adultos mayores del

Hospital Dr. Humberto Molina de Zaruma entre abril del 2015 y enero del año 2016

Level of satisfaction of the use of total bimaxillary prostheses in older adults of Dr.

Humberto Molina de Zaruma Hospital between April 2015 and January 2016

Revista Científica Mundo de la Investigación y el Conocimiento. Vol. 3 núm.1, enero,

ISSN: 2588-073X, 2019, pp. 305-328

DOI: 10.26820/recimundo/3.(1).enero.2019.305-328

URL: http://www.recimundo.com/index.php/es/article/view/372

Editorial Saberes del Conocimiento

Recibido: 20/11/2018

Aceptado: 05/01/2019

Publicado: 31/01/2019

Correspondencia: director@ recimundo.com

a. Especialista en Rehabilitación Oral; Odontólogo, Universidad de Guayaquil.

b. Especialista en Rehabilitación Oral; Odontólogo, Universidad de Guayaquil.

c. Especialista en Rehabilitación Oral; Odontólogo, Universidad de Guayaquil. 


\section{Nivel de satisfacción del uso de prótesis totales bimaxilares en adultos mayores del Hospital Dr. Humberto Molina de Zaruma entre abril del 2015 y enero del año 2016}

Vol. 3, núm. 1., (2019)

Álvaro Miguel González Ortiz; Vanessa Jomaira Veintimilla Abril; Marisela Susana Villarreal Salazar

\section{RESUMEN}

El edentulismo es una de las consecuencias de no cuidar la salud oral, este síndrome es la ausencia o la pérdida total de los dientes, sus causas pueden ser congénitas o adquiridas y estas pérdidas dentarias conllevan a alteraciones en los aspectos funcionales psicosociales y estéticos en las personas que lo padecen, por lo que el presente trabajo de investigación tendrá como objetivo establecer cuál es el nivel de satisfacción del uso de prótesis totales bimaxilares en adultos mayores del Hospital Dr. Humberto Molina de la Ciudad de Zaruma entre los meses de abril del 2015 y enero del año 2016, para lo cual se realizó un estudio observacional descriptivo, cuyo universo fueron de 90 pacientes, 36 de sexo masculino y 54 femenino, todos edéntulos totales bimaxilares, en los que se tomaron la muestra estadística, para determinar la satisfacción del paciente como consecuencia del uso de estas prótesis, se realizaron encuestas con el método GOHAI como medidas de efecto de la salud bucodental relacionada con la calidad de vida en adultos mayores. Los resultados obtenidos en la dimensión psicosocial indican que la mayoría de los pacientes se sienten satisfechos con sus prótesis dentales, en cuanto al dolor y la sensibilidad los pacientes presentaban molestias mínimas y en cuanto la dimensión física los pacientes presentaban problemas en la masticación limitándose en la clase o cantidad de alimentos la calidad de vida de los pacientes rehabilitados con prótesis totales bimaxilares en relación con la salud bucal alcanzó un nivel moderado-alto. Como conclusión podemos indicar que el efecto en las diferentes áreas de la funcionalidad bucal y los aspectos negativos relacionados con los parámetros psicosociales debida a la pérdida de dientes en los adultos mayores puede ser disminuido en gran medida por el uso de prótesis, ya que el paciente presenta una mejora de todo el funcionamiento del sistema estomagtognático y de la salud en general, cabe indicar que este grupo de población es la beneficiada de este estudio en donde se observó un aumento en su auto estima y su calidad de vida, además se estableció una mejor cultura de higiene bucal y se concientizo en la importancia del uso, mantenimiento y cambio de la prótesis, incentivando a la población a tener presente las medidas de prevención que ayuden a disminuir el edentulismo en futuras generaciones.

Palabras clave: Prótesis Dental; Adultos; GOHAI, Edentulismo. 


\title{
Nivel de satisfacción del uso de prótesis totales bimaxilares en adultos mayores del Hospital Dr. Humberto Molina de Zaruma entre abril del 2015 y enero del año 2016 \\ Vol. 3, núm. 1., (2019) \\ González, A; Ventimilla, V.; Villarreal M.
}

\begin{abstract}
Edentulism is one of the consequences of not caring for oral health, this syndrome is the absence or total loss of teeth, its causes can be congenital or acquired and these dental losses lead to alterations in the psychosocial and aesthetic functional aspects in the people who suffer from it, so this research work will aim to establish what is the level of satisfaction of the use of total bimaxillary prostheses in older adults of the Dr. Humberto Molina Hospital of the City of Zaruma between the months of April 2015 and January of 2016, for which a descriptive observational study was carried out, whose universe consisted of 90 patients, 36 of male and 54 female, all edentulous bimaxillary total, in which the statistical sample was taken, to determine the satisfaction of the patient as a consequence of the use of these prostheses, surveys with the GOHAI method were carried out as measures of the effect of oral health related to the quality of life in older adults. The results obtained in the psychosocial dimension indicate that most patients feel satisfied with their dental prostheses, in terms of pain and sensibility, patients presented minimal discomforts and in the physical dimension the patients presented problems in mastication, limited in the class or quantity of foods the quality of life of patients rehabilitated with bimaxillary total prostheses in relation to oral health reached a moderate-high level. As a conclusion we can indicate that the effect in different areas of oral functionality and the negative aspects related to psychosocial parameters due to tooth loss in older adults can be greatly diminished by the use of prosthesis, since the patient presents an improvement of the entire operation of the stomatological system and of health in general, it should be noted that this population group is the beneficiary of this study where an increase in their self-esteem and quality of life was observed, in addition a better oral hygiene culture and awareness of the importance of the use, maintenance and change of the prosthesis, encouraging the population to keep in mind the prevention measures that help reduce edentulism in future generations.
\end{abstract}

Keywords: Dental Prosthesis; Adults; GOHAI, Edentulism. 


\section{Nivel de satisfacción del uso de prótesis totales bimaxilares en adultos mayores del Hospital Dr. Humberto Molina de Zaruma entre abril del 2015 y enero del año 2016}

Vol. 3, núm. 1., (2019)

Álvaro Miguel González Ortiz; Vanessa Jomaira Veintimilla Abril; Marisela Susana Villarreal Salazar

\section{Introducción.}

En la segunda mitad del siglo pasado, la población ecuatoriana mejoró su esperanza de vida, pasó de 48,3 años en 1950-55 a 75,6 años en 2010-15 esto permitió que muchos ecuatorianos aumentaran su calidad de vida y alcanzaran edades mayores.

Numerosos estudios han demostrado que la mayoría de los adultos mayores tienen problemas de masticación, dolor, dificultades al comer, y problemas en las relaciones sociales a causa de los trastornos bucales; la condición de mayor prevalencia es la ausencia de todos los dientes o edentulismo. (D.Locker, Clarke, \& B.Payne, 2000).

Según: (Von, 2015) en su artículo sobre el "Rendimiento masticatorio y nivel de satisfacción de pacientes tratados con prótesis totales en la "Universidad Mayor" en donde se aplicó la encuesta GOHAI a 18 pacientes rehabilitados con prótesis totales bimaxilares para conocer el nivel de satisfacción con el uso de sus prótesis. Los pacientes portadores de prótesis totales señalaron un bajo nivel de satisfacción con el uso de sus dentaduras completas, en especial durante la función masticatoria. (Von, 2015)

El mayor factor de inconformidad se manifiesta con la masticación, y en particular con cierto tipo de alimentos; esta condición obliga a muchos de estos pacientes a seleccionar y restringir muchos alimentos de su dieta, generando disgusto y menoscabo, sobre todo cuando se está en presencia de otros individuos. (Von, 2015) 


\section{Nivel de satisfacción del uso de prótesis totales bimaxilares en adultos mayores del Hospital Dr. Humberto Molina de Zaruma entre abril del 2015 y enero del año 2016 \\ Vol. 3, núm. 1., (2019) \\ González, A; Ventimilla, V.; Villarreal M.}

El análisis de la dimensión psicosocial de la encuesta GOHAI mostró que un gran número de pacientes estaban satisfechos con su estética y que sus relaciones sociales mejoraron después de su tratamiento protésico protésico. (Von, 2015)

Según estudios realizados en Cartagena de Indias, cuyos sujetos de estudio fueron 120 pacientes adultos mayores de 60 años para la identificación del impacto de la salud oral en la calidad de vida de los adultos mayores, los resultados demostraron que; al analizar las dimensiones evaluadas por el (GOHAI), que relacionan la salud bucal con la calidad de vida. (Díaz, Arrieta, \& Martínez, 2012)

Se encontró que la función psicosocial fue el componente reportado con mayor frecuencia, en especial aquél relacionado con la incomodidad que presentan los adultos mayores al comer frente a otras personas por problemas con sus dientes (44\%), con respecto a la función física, el 63,3\% manifestó incomodidad al deglutir sus alimentos y, con respecto a la sensibilidad dental, la molestia más percibida fue la sensibilidad a los alimentos calientes, fríos o dulces, en un 42,5\%. (Díaz, Arrieta, \& Martínez, 2012)

\section{Edentulismo total aspectos generales y demográficos}

El edentulismo se define como la ausencia total de piezas dentales, las cuales no se remplazarán posteriormente de forma natural. (Sánchez, Román, Dávila, \& González, 2011)

El edentulismo se observa con mayor frecuencia en grupos de nivel socioeconómico bajo, en donde la principal causa de pérdida dental viene a ser la enfermedad periodontal, caries, el trauma y la iatrogenia. Posteriormente se desencadena una sobrecarga en los dientes remanentes, 


\section{Nivel de satisfacción del uso de prótesis totales bimaxilares en adultos mayores del Hospital Dr. Humberto Molina de Zaruma entre abril del 2015 y enero del año 2016}

Vol. 3, núm. 1., (2019)

Álvaro Miguel González Ortiz; Vanessa Jomaira Veintimilla Abril; Marisela Susana Villarreal Salazar

provocando movilidad dentaria y alteraciones oclusal, reabsorción alveolar y edentulismo total.

(Ozawa, Fundamentos de prostodoncia total., 2010)

La pérdida de dientes determina cambios morfológicos, funcionales, psicológicos y sociales que se debe tener en consideración el momento de tratar pacientes de la tercera edad, ya que si existe un conocimiento insuficiente de estos aspectos se pone en riesgo el tratamiento con prótesis completas, produciendo complicaciones o lesiones en el sistema estomatognático. (Koeck, 2007)

El Informe Estadístico sobre la Salud en el Mundo de la OMS, señala que el más alto de los valores promedio de la edad de vida en la gente está en Latinoamérica, donde se rebasa los 70 años promedio en forma notoria. Ecuador está por ello en el plano medio de esta calificación con una expectativa de vida a los 73 años de edad. La esperanza de vida en América del Norte y del Sur es la más alta del mundo. (OMS, 2014)

\section{Cambios en los tejidos periodontales asociados al envejecimiento}

El adulto mayor es una persona que como los demás grupos edad, se está adaptando a una serie de cambios socioculturales que le llevan a disfrutar de una mejor calidad de vida, por lo menos en las sociedades más avanzadas y a lo mejor, en menor escala en las menos desarrolladas. Esta situación, genera un aumento en la incidencia y prevalencia de la enfermedad periodontal dentro de la población mayor. (Huttner, Machado, Oliveira, Antunes, \& Hebling, 2009) 


\section{Nivel de satisfacción del uso de prótesis totales bimaxilares en adultos mayores del Hospital Dr. Humberto Molina de Zaruma entre abril del 2015 y enero del año 2016 \\ Vol. 3, núm. 1., (2019) \\ González, A; Ventimilla, V.; Villarreal M.}

Clásicamente se creía que la pérdida de inserción epitelial y el hueso alveolar eran cambios periodontales relacionados con la edad, pero realmente estos hechos no se corresponden con el fenómeno del envejecimiento propiamente dicho. Y más aun teniendo en la actualidad tan claro el origen y factores determinantes en el proceso de la enfermedad periodontal. (Huttner, Machado, Oliveira, Antunes, \& Hebling, 2009)

Pero aunque es difícil separar los condicionantes fisiológicos y patológicos en el envejecimiento periodontal, si se consideran dentro de las características más relevantes en los ancianos la retracción o recesión gingival, donde hay una importante combinación de factores irritativos y biológicos de tipo bacteriano, y a la vez factores traumáticos como el excesivo e incorrecto cepillado (Huttner, Machado, Oliveira, Antunes, \& Hebling, 2009)

\section{Hueso Alveolar}

La reabsorción ósea del maxilar y la mandíbula, en el adulto mayor produce uno de los cambios estructurales más importantes como es la aparición de un reborde residual agudo, así como también la aproximación del reborde residual al agujero mentoniano. (Ozawa, Fundamentos de prostodoncia total., 2010)

De esta manera, el hueso maxilar dificulta la estabilidad de la base protésica inferior; y en caso de reabsorción extrema se debe considerar que el paciente puede sufrir fracturas mandibulares en la edad avanzada. La rama ascendente aparece relativamente larga, al reducirse la altura del cuerpo mandibular. (Ozawa, Fundamentos de prostodoncia total., 2010) 


\section{Nivel de satisfacción del uso de prótesis totales bimaxilares en adultos mayores del Hospital Dr. Humberto Molina de Zaruma entre abril del 2015 y enero del año 2016}

Vol. 3, núm. 1., (2019)

Álvaro Miguel González Ortiz; Vanessa Jomaira Veintimilla Abril; Marisela Susana Villarreal Salazar

Los fenómenos de reabsorción ósea que sufre el maxilar son la reabsorción centrípeta y la reabsorción vertical provocando cambios en los tejidos blandos ya que ellos acompañan el proceso de reabsorción, determinando la disminución del tono muscular, acentuando las líneas de expresión y acelerando las características de envejecimiento. (Pietrokovski, Starinsky, Arensburg, \& Kaffe, 2007)

El resultado de esta reabsorción es un pseudoprognatismo mandibular y retrusión maxilar (patrón esqueletal pseudo clase III), también suele obligar a una mordida cruzada posterior de la prótesis para mantenerla sobre los rebordes residuales, lo que en adultos mayores se observa con mayor frecuencia debido al edentulismo. (Rahn, Ivanhoe, \& Plummer, Prótesis dental completa, 2011)

\section{Mucosa Oral}

En la mucosa bucal existe atrofia de epitelio, disminución de la queratinización, disminución de la cantidad de células en tejido conectivo, aumento de sustancias intercelular y descenso del consumo de oxígeno; la falta de elasticidad con resequedad y atrofia, tiende al hiperqueratosis. (Habeest, Brass, \& Merkesteim, 1998)

Experimenta cambios relacionados con factores locales adquiridos a lo largo de la vida como la dieta, el hábito de fumar, el alcoholismo y la prótesis, volviéndose más delgada, lisa y seca, tornándose permeable a sustancias nocivas y más propensas a daños mecánicos. (Habeest, Brass, \& Merkesteim, 1998) 


\section{Nivel de satisfacción del uso de prótesis totales bimaxilares en adultos mayores del Hospital Dr. Humberto Molina de Zaruma entre abril del 2015 y enero del año 2016 \\ Vol. 3, núm. 1., (2019) \\ González, A; Ventimilla, V.; Villarreal M.}

La pérdida excesiva del espesor de la mucosa aumenta la susceptibilidad a procesos inflamatorios acelerando la reabsorción ósea. También conduce a la formación de fibroma gingival, que depende del tiempo y de la magnitud de las cargas, que es una irritación provocada por los márgenes demasiado extensos y afilados de la prótesis. (Koeck, 2007)

\section{Cambios psicológicos y sociales}

En cuanto a los cambios psicológicos podemos decir que el edentulismo total, transforma el contorno facial, altera el lenguaje, dificulta la alimentación, modifica la nutrición, altera la expresión y repercute en la mente y en los sentimientos. En estos pacientes existen cambios no solo estructurales y biológicos, sino que también aparecen notables trastornos o cambios psicológicos como desórdenes de la conducta, problemas en relación con la fuerza del ego y la autoestima. (Ozawa, Prostodoncia total, 1995)

El desmoronamiento del ego se manifiesta por dos vías: la llamada reacción depresiva, que es el desorden psíquico más común que presentan los ancianos, y consiste en una percepción de cambios en su estado físico o social que él reconoce como una amenaza a la consideración de sí mismo, pero no hay sensación de culpa u hostilidad interna en estos pacientes; y la llamada demencia senil, cuando sufren de cambios orgánicos en el sistema nervioso central con arteriosclerosis cerebral, presentan patrones de conducta perturbados. (Ozawa, Prostodoncia total, 1995)

En general son hostiles, sospechan mucho de las motivaciones del odontólogo y discuten con exceso, tienen dificultad para adaptarse a cualquier problema, les falta voluntad para 


\section{Nivel de satisfacción del uso de prótesis totales bimaxilares en adultos mayores del Hospital Dr. Humberto Molina de Zaruma entre abril del 2015 y enero del año 2016}

Vol. 3, núm. 1., (2019)

Álvaro Miguel González Ortiz; Vanessa Jomaira Veintimilla Abril; Marisela Susana Villarreal Salazar

cooperar y han perdido el deseo de adaptarse a nuevas realidades. Al resultado de todos estos factores tan variados $\mathrm{y}$ diferentes se le denomina "envejecimiento mental normal" $\mathrm{u}$ ortoinvolución, durante el cual tiene lugar un deterioro gradual de las diferentes facultades. (Ozawa, Prostodoncia total, 1995)

En personas edéntulas totales los aspectos psicológicos pueden variar de mínimo hasta en algunos casos llegar a un estado de neurosis; con pacientes que consideran que las prótesis satisfacen las necesidades tanto en masticación y estética, pero otros indican que su vida social es considerablemente afectada, porque pierden la confianza en sí mismo por lo tanto sienten vergüenza. (Misch, 2009)

\section{Estética}

La evaluación clínica de la estética que proporciona la prótesis al paciente nos permite confirmar el estado de una prótesis total y observar si el aspecto facial del paciente mejora o no con el uso de la misma. De esta manera podemos observar el color, forma, tamaño de los dientes, orientación y nivel del plano oclusal, posición de la línea media, grado de soporte labial y desgaste oclusal. (Basker, Davenport, \& Thomason, 2012)

\section{Confort}

Antes de insertar las prótesis nuevas por primera vez, debe examinarse cuidadosamente la superficie de impresión por cualquier causa potencial de dolor, si está presente, debe ser eliminado, para evitar la adopción de trayectorias de cierre anormales de la mandíbula, que 


\section{Nivel de satisfacción del uso de prótesis totales bimaxilares en adultos mayores del Hospital Dr. Humberto Molina de Zaruma entre abril del 2015 y enero del año 2016 \\ Vol. 3, núm. 1., (2019) \\ González, A; Ventimilla, V.; Villarreal M.}

puede seguirse para evitar la presión oclusal en el sitio del malestar. (Basker, Davenport, \& Thomason, 2012)

Debe asegurarse que los dietes ocluyan uniformemente, que las prótesis permanezcan en su lugar cuando sean insertadas, durante la apertura normal de la boca, al hablar, comer, y de esta manera garantizar el confort del paciente. Esto determina que una prótesis dental debe garantizar ese normal desempeño de manera cómoda sin generar mayores traumatismos a los que ya ha sido expuesta la boca. (Basker, Davenport, \& Thomason, 2012)

\section{Calidad de vida y salud bucal}

En la literatura científica, se vienen realizando diferentes estudios y aplicaciones de la calidad de vida asociada al estado bucodental, tema que día tras día viene tomando más importancia en el mundo. El concepto de calidad de vida viene cubriendo cada vez más páginas de las revistas odontológicas, ya que es justificado su uso en la evaluación de estados de salud, grupos de riesgo, objetivos y predicciones de tratamientos, y todos se justifican por la finalidad de tener mejoras en la calidad de vida de los pacientes. (Cunningham \& Hunt, 2002)

Los estudios clínicos quieren relacionar la calidad de vida y la salud del individuo, o sea estimar la calidad de vida como una propuesta para evaluar los cuidados de la salud, de esta manera es que se vienen presentando de forma cada vez más frecuentes las publicaciones de cerca 1000 artículos anualmente con el término calidad de vida. (Cunningham \& Hunt, 2002)

Calidad de vida la definen como la medida en que se modifica el valor asignado a la duración de la vida en función de la percepción de limitaciones físicas, psicológicas, sociales y de 


\section{Nivel de satisfacción del uso de prótesis totales bimaxilares en adultos mayores del Hospital Dr. Humberto Molina de Zaruma entre abril del 2015 y enero del año 2016}

Vol. 3, núm. 1., (2019)

Álvaro Miguel González Ortiz; Vanessa Jomaira Veintimilla Abril; Marisela Susana Villarreal Salazar

disminución de oportunidades a causa de la enfermedad, sus secuelas, el tratamiento y/o las políticas de salud. (Patrick \& Erickson, 1993)

\section{Geriatric Oral HealthAssessmentIndex - GOHAI}

En 1990, los doctores(as) Kathryn A. Atchison, y Teresa A. Dolan del departamento de salud pública odontológica de la universidad de California en los Ángeles (EU), tras los estudios e investigaciones con adultos mayores norteamericanos desarrollaron y publicaron en el "Journal of Dental Education" el "Geriatric Oral Health Assessment Index" traducido como "Índice de Calidad de Vida Oral en Geriatría” (GOHAI) ó "Índice de Valoración de salud Oral en Geriatria. (Atchison \& Dolan, 1990)

Esta herramienta fue diseñada para valorar en poblaciones de adultos mayores, los problemas oro-funcionales y estimar grado de impacto psicosocial asociado a los problemas bucodentales ó también para evaluar la efectividad funcional y psicosocial de un tratamiento bucodental realizado. (Atchison \& Dolan, 1990)

El GOHAI está compuesto por 12 ítems o preguntas y evalúa tres dimensiones:

1. Función física, que comprende aspectos como comer, hablar, y tragar.

2. Función psicosocial, comprende aspectos de preocupación acerca de la salud oral, autoimagen, conciencia de la salud y limitación de los contactos sociales por causa de problemas orales.

3. Dolor o incomodidad asociados al estado bucodental. (Pinzon-Pulido \& Gil-Montoya, 1999) 


\section{Nivel de satisfacción del uso de prótesis totales bimaxilares en adultos mayores del Hospital Dr. Humberto Molina de Zaruma entre abril del 2015 y enero del año 2016 \\ Vol. 3, núm. 1., (2019) \\ González, A; Ventimilla, V.; Villarreal M.}

La respuesta a las variables o ítems, es obtenido con una escala de Likert con 5 niveles de respuesta:

$\mathrm{S}=$ siempre (1); $\mathrm{F}=$ frecuentemente (2); $\mathrm{AV}=$ algunas veces (3); $\mathrm{RV}=$ rara vez (4); $\mathrm{N}=$ nunca (5).

Los ítems 2 y 10 tienen una valoración inversa al resto: donde, siempre $=5$; frecuentemente $=4$; algunas veces $=3$; rara vez $=2$; nunca $=1$, conversión que se realiza al momento del análisis. (Atchison \& Dolan, 1990)

El valor total del GOHAI, se establece sumando las respuestas de los 12 ítems, pudiendo variar de cero a sesenta. Las escalas del GOHAI fueron divididas en tres categorías: alto, moderado y bajo nivel de salud. Una escala de GOHAI de 57 a 60 es considerada como nivel alto, de 51 a 56 como moderado, y 50 o menor valor es considerado como nivel bajo. (Atchison \& Dolan, 1990)

\section{Adultos mayores en el Ecuador}

\section{Estado de la situación de las personas adultas mayores}

En el Ecuador hay 14`483.499 habitantes y de ellos 940.905 personas son mayores de 65 años, es decir el 6,5 \% (INEC, Censo de Población y Vivienda 2010). A nivel nacional existe una diferencia de cinco puntos entre mujeres y hombres (53 a 47\%, respectivamente), siendo siempre mayor el número de mujeres en los diferentes grupos de edad. Las provincias de Manabí y Azuay son las que tienen mayor cantidad de personas adultas mayores que superan las 90.000 personas. (MIES, 2013) 
Nivel de satisfacción del uso de prótesis totales bimaxilares en adultos mayores del Hospital Dr. Humberto Molina de Zaruma entre abril del 2015 y enero del año 2016

Vol. 3, núm. 1., (2019)

Álvaro Miguel González Ortiz; Vanessa Jomaira Veintimilla Abril; Marisela Susana Villarreal Salazar

\section{Condiciones socioeconómicas}

De acuerdo a la información censal (INEC. Censo de Población y Vivienda 2010), las condiciones socioeconómicas en las que viven los adultos mayores en nuestro país son en su mayoría deficitarias, estimándose que el 57,4\%, corresponde a 537.421 personas, se encuentra en condiciones de pobreza y extrema pobreza. (MIES, 2013)

Resultados y análisis.

Tabla $N^{\circ} 1$

\begin{tabular}{|c|c|c|}
\hline GÉNERO & $\mathbf{N}^{\circ}$ PACIENTES & PORCENTAJE \\
\hline MASCULINO & 36 & 40 \\
\hline FEMENINO & 54 & 60 \\
\hline TOTAL & $\mathbf{9 0}$ & $\mathbf{1 0 0}$ \\
\hline
\end{tabular}

\section{Objetivo 1}

Identificar los aspectos psicosociales relacionados con el uso de las prótesis totales bimaxilares.

1. ¿Evitó estar en contacto con personas debido a la condición de su prótesis dental?

Tabla $\mathrm{N}^{\circ} 2$

\begin{tabular}{|c|c|c|}
\hline OPCIONES & FRECUENCIA & \% \\
\hline SIEMPRE & 6 & 7 \\
\hline FRECUENTEMENTE & 12 & 13 \\
\hline A VECES & 16 & 18 \\
\hline RARA VEZ & 22 & 24 \\
\hline NUNCA & 34 & 38 \\
\hline TOTAL & $\mathbf{9 0}$ & $\mathbf{1 0 0}$ \\
\hline
\end{tabular}

Revista Científica Mundo de la Investigación y el Conocimiento. 3 (1). pp. 305-328 
Nivel de satisfacción del uso de prótesis totales bimaxilares en adultos mayores del Hospital Dr. Humberto Molina de Zaruma entre abril del 2015 y enero del año 2016

Vol. 3, núm. 1., (2019) González, A; Ventimilla, V.; Villarreal M.

2. ¿Se sintió satisfecho o contento con la apariencia de su prótesis dental?

Tabla $\mathrm{N}^{\circ} 3$

\begin{tabular}{|c|c|c|}
\hline OPCIONES & FRECUENCIAS & \% \\
\hline SIEMPRE & 49 & 54 \\
\hline FRECUENTEMENTE & 19 & 21 \\
\hline A VECES & 14 & 16 \\
\hline RARA VEZ & 5 & 6 \\
\hline NUNCA & 3 & 3 \\
\hline TOTAL & $\mathbf{9 0}$ & $\mathbf{1 0 0}$ \\
\hline
\end{tabular}

3. ¿Cuántas veces se preocupó o intranquilizo por su prótesis dental?

Tabla $\mathrm{N}^{\circ} 4$

\begin{tabular}{|c|c|c|}
\hline OPCIONES & FRECUENCIAS & \% \\
\hline SIEMPRE & 8 & 9 \\
\hline FRECUENTEMENTE & 16 & 18 \\
\hline A VECES & 17 & 19 \\
\hline RARA VEZ & 22 & 24 \\
\hline NUNCA & 27 & 30 \\
\hline TOTAL & $\mathbf{9 0}$ & $\mathbf{1 0 0}$ \\
\hline
\end{tabular}

4. ¿Se sintió nervioso o consiente debido a problemas con su prótesis dental?

Tabla $\mathrm{N}^{\circ} 5$

\begin{tabular}{|c|c|c|}
\hline OPCIONES & FRECUENCIAS & $\mathbf{\%}$ \\
\hline SIEMPRE & 12 & 13 \\
\hline FRECUENTEMENTE & 14 & 15 \\
\hline
\end{tabular}


Nivel de satisfacción del uso de prótesis totales bimaxilares en adultos mayores del Hospital Dr. Humberto Molina de Zaruma entre abril del 2015 y enero del año 2016

Vol. 3, núm. 1., (2019)

Álvaro Miguel González Ortiz; Vanessa Jomaira Veintimilla Abril; Marisela Susana Villarreal Salazar

\begin{tabular}{|c|c|c|}
\hline A VECES & 21 & 23 \\
\hline RARA VEZ & 17 & 18 \\
\hline NUNCA & 26 & 28 \\
\hline TOTAL & $\mathbf{9 0}$ & $\mathbf{1 0 0}$ \\
\hline
\end{tabular}

5. ¿Se sintió incomodo al comer frente a otras personas debido a su prótesis dental? Tabla $\mathrm{N}^{\circ} 6$

\begin{tabular}{|c|c|c|}
\hline OPCIONES & FRECUENCIAS & \% \\
\hline SIEMPRE & 4 & 4 \\
\hline FRECUENTEMENTE & 9 & 10 \\
\hline A VECES & 14 & 15 \\
\hline RARA VEZ & 29 & 32 \\
\hline NUNCA & 34 & 37 \\
\hline TOTAL & $\mathbf{9 0}$ & $\mathbf{1 0 0}$ \\
\hline
\end{tabular}

6. ¿Evito reírse o sonreírse debido a problemas estéticos en su prótesis dental?

Tabla $\mathrm{N}^{\circ} 7$

\begin{tabular}{|c|c|c|}
\hline OPCIONES & FRECUENCIAS & $\mathbf{\%}$ \\
\hline SIEMPRE & 9 & 9 \\
\hline FRECUENTEMENTE & 10 & 10 \\
\hline A VECES & 18 & 19 \\
\hline RARA VEZ & 22 & 24 \\
\hline NUNCA & 32 & 35 \\
\hline TOTAL & $\mathbf{9 1}$ & $\mathbf{1 0 0}$ \\
\hline
\end{tabular}


Nivel de satisfacción del uso de prótesis totales bimaxilares en adultos mayores del Hospital Dr. Humberto Molina de Zaruma entre abril del 2015 y enero del año 2016

Vol. 3, núm. 1., (2019) González, A; Ventimilla, V.; Villarreal M.

\section{Objetivo 2}

Analizar cuáles son las causas que provocan dolor durante el uso de la prótesis total

7. ¿Ha tenido dolor o molestias alrededor o dentro de la boca?

Tabla $N^{\circ} 8$

\begin{tabular}{|c|c|c|}
\hline OPCIONES & FRECUENCIAS & $\mathbf{\%}$ \\
\hline SIEMPRE & 11 & 12 \\
\hline FRECUENTEMENTE & 12 & 13 \\
\hline A VECES & 17 & 18 \\
\hline RARA VEZ & 23 & 25 \\
\hline NUNCA & 27 & 30 \\
\hline TOTAL & $\mathbf{9 0}$ & $\mathbf{1 0 0}$ \\
\hline
\end{tabular}

8. ¿Sintió sus encías sensibles a los alimentos calientes, fríos o dulces?

Tabla $\mathrm{N}^{\circ} 9$

\begin{tabular}{|c|c|c|}
\hline OPCIONES & FRECUENCIAS & $\mathbf{\%}$ \\
\hline SIEMPRE & 7 & 8 \\
\hline FRECUENTEMENTE & 12 & 13 \\
\hline A VECES & 20 & 22 \\
\hline RARA VEZ & 22 & 24 \\
\hline NUNCA & 29 & 32 \\
\hline TOTAL & $\mathbf{9 0}$ & $\mathbf{1 0 0}$ \\
\hline
\end{tabular}


Nivel de satisfacción del uso de prótesis totales bimaxilares en adultos mayores del Hospital Dr. Humberto Molina de Zaruma entre abril del 2015 y enero del año 2016

Vol. 3, núm. 1., (2019)

Álvaro Miguel González Ortiz; Vanessa Jomaira Veintimilla Abril; Marisela Susana Villarreal Salazar

\section{Objetivo 3}

Establecer los aspectos físicos relacionados con el uso de las prótesis totales bimaxilares.

9. ¿Limitó la clase o cantidad de alimentos debido a su prótesis dental?

Tabla $\mathrm{N}^{\circ} 10$

\begin{tabular}{|c|c|c|}
\hline & & \\
OPCIONES & FRECUENCIAS & $\mathbf{\%}$ \\
\hline SIEMPRE & 14 & 15 \\
\hline FRECUENTEMENTE & 24 & 25 \\
\hline A VECES & 16 & 17 \\
\hline RARA VEZ & 21 & 22 \\
\hline NUNCA & 20 & 21 \\
\hline TOTAL & $\mathbf{9 0}$ & $\mathbf{1 0 0}$ \\
\hline
\end{tabular}

10. ¿Cuántas veces pudo tragar cómodamente?

Tabla $\mathrm{N}^{\circ} 11$

\begin{tabular}{|c|c|c|}
\hline OPCIONES & FRECUENCIAS & $\mathbf{\%}$ \\
\hline SIEMPRE & 33 & 37 \\
\hline FRECUENTEMENTE & 26 & 29 \\
\hline A VECES & 15 & 17 \\
\hline RARA VEZ & 9 & 10 \\
\hline NUNCA & 7 & 8 \\
\hline TOTAL & $\mathbf{9 0}$ & $\mathbf{1 0 0}$ \\
\hline
\end{tabular}

11. ¿Su prótesis dental le impidió hablar del modo que usted quería?

Tabla $\mathrm{N}^{\circ} 12$ 
Nivel de satisfacción del uso de prótesis totales bimaxilares en adultos mayores del Hospital Dr. Humberto Molina de Zaruma entre abril del 2015 y enero del año 2016

Vol. 3, núm. 1., (2019) González, A; Ventimilla, V.; Villarreal M.

\begin{tabular}{|c|c|c|}
\hline OPCIONES & FRECUENCIAS & \% \\
\hline SIEMPRE & 16 & 18 \\
\hline FRECUENTEMENTE & 15 & 17 \\
\hline A VECES & 18 & 20 \\
\hline RARA VEZ & 21 & 24 \\
\hline NUNCA & 20 & 23 \\
\hline TOTAL & $\mathbf{9 0}$ & $\mathbf{1 0 0}$ \\
\hline
\end{tabular}

12. ¿Pudo comer lo que usted quería sin tener ninguna molestia de su prótesis dental?

Tabla $\mathrm{N}^{\circ} 13$

\begin{tabular}{|c|c|c|}
\hline OPCIONES & FRECUENCIAS & \% \\
\hline SIEMPRE & 26 & 29 \\
\hline FRECUENTEMENTE & 21 & 24 \\
\hline A VECES & 19 & 22 \\
\hline RARA VEZ & 10 & 12 \\
\hline NUNCA & 14 & 16 \\
\hline TOTAL & $\mathbf{9 0}$ & $\mathbf{1 0 0}$ \\
\hline
\end{tabular}


Nivel de satisfacción del uso de prótesis totales bimaxilares en adultos mayores del Hospital Dr. Humberto Molina de Zaruma entre abril del 2015 y enero del año 2016

Vol. 3, núm. 1., (2019)

Álvaro Miguel González Ortiz; Vanessa Jomaira Veintimilla Abril; Marisela Susana Villarreal Salazar

\section{ESCALA LIKERT-GOHAI-VALORES TOTALES}

\begin{tabular}{|c|c|c|c|c|c|}
\hline VALOR & 1 & 2 & 3 & 4 & 5 \\
\hline \multicolumn{2}{|c|}{ PREGUNTAS SIEMPRE } & FRECUEN & A VECES & RARA VEZ & NUNCA \\
\hline 1 & 6 & 12 & 16 & 22 & 34 \\
\hline 3 & 8 & 16 & 17 & 22 & 27 \\
\hline 4 & 12 & 14 & 21 & 17 & 26 \\
\hline 5 & 4 & 9 & 14 & 29 & 34 \\
\hline 6 & 9 & 10 & 18 & 22 & 32 \\
\hline 7 & 11 & 12 & 17 & 23 & 27 \\
\hline 8 & 7 & 12 & 20 & 22 & 29 \\
\hline 9 & 12 & 18 & 16 & 18 & 26 \\
\hline 11 & 13 & 15 & 18 & 21 & 23 \\
\hline \multirow[t]{2}{*}{12} & 26 & 21 & 19 & 10 & 14 \\
\hline & $108 \times 1$ & $139 \times 2$ & $176 \times 3$ & 206X4 & $272 \times 5$ \\
\hline TOTAL & 108 & 278 & 528 & 824 & 1360 \\
\hline VALOR & 5 & 4 & 3 & 2 & 1 \\
\hline \multicolumn{2}{|c|}{ PREGUNTAS SIEMPRE } & FRECUEN & A VECES & RARA VEZ & NUNCA \\
\hline 2 & 49 & 19 & 14 & 5 & 3 \\
\hline \multirow[t]{2}{*}{10} & 33 & 26 & 15 & 9 & 7 \\
\hline & $82 \times 5$ & $45 \times 4$ & $29 \times 3$ & $14 \times 2$ & $10 \times 1$ \\
\hline TOTAL & 410 & 180 & 87 & 28 & 10 \\
\hline TOTAL & 518 & 458 & 615 & 852 & 1370 \\
\hline TOTAL & 3813 & & & & \\
\hline
\end{tabular}




\section{Nivel de satisfacción del uso de prótesis totales bimaxilares en adultos mayores del Hospital Dr. Humberto Molina de Zaruma entre abril del 2015 y enero del año 2016 \\ Vol. 3, núm. 1., (2019) \\ González, A; Ventimilla, V.; Villarreal M.}

\section{Discusión.}

Los resultados encontrados en el presente trabajo respecto al nivel de satisfacción que relaciona la salud oral con la calidad de vida medido por el GOHAI. En las preguntas que tienen relación y evalúan la parte psicosocial de los adultos mayores los resultados indican que la mayoría de los pacientes se sienten satisfechos con sus prótesis dentales estos resultados son similares a los reportados en las publicaciones realizadas por Daniela Von Kretschmann et al en su trabajo de investigación sobre el rendimiento masticatorio y el nivel de satisfacción de pacientes tratados con prótesis totales en la universidad mayo.

En cuanto al dolor los resultados más relevantes fueron cuando los pacientes presentaban molestias mínimas en la boca estos resultados presentan porcentajes mayores en relación con los datos reportados por Daniela Von Kretschmann en Chile en su estudio sobre el Rendimiento masticatorio y nivel de satisfacción de pacientes tratados con prótesis totales en la Universidad Mayor, donde reporta que más de la mitad de los pacientes nunca tienen problemas con el dolor o la incomodidad con el uso de sus prótesis.

Con respecto a la dimensión física los resultados más relevantes encontrados fueron que los individuos tuvieron limitaciones en cuanto a la calidad y cantidad de alimentos, eligiendo los más blandos, estos resultados son similares a los reportados en otros estudios para adultos mayores antes mencionados. 


\section{Nivel de satisfacción del uso de prótesis totales bimaxilares en adultos mayores del Hospital Dr. Humberto Molina de Zaruma entre abril del 2015 y enero del año 2016}

Vol. 3, núm. 1., (2019)

Álvaro Miguel González Ortiz; Vanessa Jomaira Veintimilla Abril; Marisela Susana Villarreal Salazar

\section{Conclusiones.}

Con este trabajo de investigación se pudo diagnosticar y determinar los parámetros de la salud oral que influyen en la calidad de vida de los pacientes adultos mayores de bajos recursos económicos atendidos del Hospital Dr. Humberto Molina de Zaruma.

Los pacientes edéntelos bimaxilares presentan problemas gástricos, trastornos psicológicos y niveles bajos de autoestima debido a la pérdida de sus dientes a temprana edad y por no tener dinero para hacerse una prótesis.

La mayor valoración que se presenta utilizando el índice GOHAI hace referencia a los aspectos psicológicos en donde se observa una gran aceptación de las prótesis por parte de los pacientes.

Los niveles de satisfacción en cuanto a la función estética, física y del dolor, alcanzan las expectativas de los pacientes.

La encuesta GOHAI es válida como un método para medir la calidad de vida del paciente en relación con su salud oral.

El uso de las prótesis totales bimaxilares influyo directamente mejorando la calidad de vida en los pacientes adultos mayores atendidos en el Hospital de Zaruma. 


\section{Nivel de satisfacción del uso de prótesis totales bimaxilares en adultos mayores del Hospital Dr. Humberto Molina de Zaruma entre abril del 2015 y enero del año 2016 \\ Vol. 3, núm. 1., (2019) \\ González, A; Ventimilla, V.; Villarreal M.}

\section{Bibliografía.}

Atchison, K., \& Dolan, T. (Noviembre de 1990). Development of the Geriatric Oral Health Assessment Index. Journal of Dental Education, 14(11), 680-7.

Basker, R., Davenport, J., \& Thomason, J. (2012). Tratamiento protésico en pacientes edentulos. The United Kingdong: Amolca, Actualidades Medicas CA.

Cunningham, S. G., \& Hunt, N. (2002). Development of a condition-specific quality of life measure for patients with dentofacial deformity y: II. Validity and. Community Dent Oral Epidemiol., 81-90.

D.Locker, Clarke, M., \& B.Payne. (2000). Self-perceived oral health status, psychological wellbeing, and life satisfaction in an older adult population. $J$ Dent Res, 970-5.

Díaz, S., Arrieta, K., \& Martínez, K. R. (Febrero de 2012). Impacto de la salud oral en la calidad de vida de adultos mayores. Cartagena, Colombia.

Habeest, L., Brass, J., \& Merkesteim, J. (1998). Mandibular atrophy and metabolic bone loss.Histomorphometry of ilioc crest biopsies in 24 patients. J Oral Maxilofac Surg, 3259.

Huttner, E., Machado, D., Oliveira, R., Antunes, A., \& Hebling, E. (2009). Effects of human aging on periodontal tissues. Spec Care Dentist, 149-55.

Koeck, B. (2007). Prótesis completas. Barcelona . Barcelona: Elsevier Doyma, S.L.

MIES. (2013). Obtenido de http://www.inclusion.gob.ec/wpcontent/uploads/downloads/2012/09/Agendas_ADULTOS.pdf

Misch, C. (2009). Implantología Contemporánea. España: Elsevier.

OMS. (2014). Ginebra Suiza: Oms.

Ozawa, J. (1995). Prostodoncia total. Mexico: Universidad nacional autonoma de mexico.

Ozawa, J. (2010). Fundamentos de prostodoncia total. Mexico: Trillas.

Patrick, D., \& Erickson, P. (1993). Health Policy, Quality of Life: Health Care Evaluation and Resource Allocation. . Oxford University Press.

Pietrokovski, J., Starinsky, R., Arensburg, B., \& Kaffe, I. (2007). Morphologic Characteristics of Bony Edentulous Jaws. journal of prosthodontic, 44.

Revista Científica Mundo de la Investigación y el Conocimiento. 3 (1). pp. 305-328 


\section{Nivel de satisfacción del uso de prótesis totales bimaxilares en adultos mayores del Hospital Dr. Humberto Molina de Zaruma entre abril del 2015 y enero del año 2016}

Vol. 3, núm. 1., (2019)

Álvaro Miguel González Ortiz; Vanessa Jomaira Veintimilla Abril; Marisela Susana Villarreal Salazar

Pinzon-Pulido, S., \& Gil-Montoya, J. (1999). Validación del Índice de Valoración en Salud Oral en Geriatría en una población Geriátrica Institucionalizada de Granada. Rev Esp Geriatr y Gerontol., 273-82.

Rahn, A., Ivanhoe, J., \& Plummer, K. (2011). Prótesis dental completa. Buenos Aires: Editorial Médica Panamericana, S.A.C.F. .

Sánchez, M., Román, M., Dávila, R., \& González, A. (2011). Salud bucal en pacientes adultos mayores y su asociación con la calidad de vida. Revista de Especialidades MédicoQuirúrgicas, No.2, 111.

Von, D. e. (2015). rendimiento masticatorio y nivel de satisfaccion y pacientes tratados con protesis totales en la universidad mayo. Clinica de Periodoncia implantologia y rehabilatacion oral, 17-23. 